\title{
Uber Jacksonsche Krämpfe mit tonischem Beginn und über ein kleines Angiocavernom des Gehirns.
}

Von

\author{
M. Lewandowsky und F. Selberg. \\ (Aus der chirurgischen Abteilung des Auguste Viktoria-Krankenhauses \\ vom Roten Kreuz zu Berlin-Weißensee.)
}

Mit 1 Tafel.

(Eingegangen am 28. Juli 1913.)

Der nachfolgende Fall wird aus zwei Gründen mitgeteilt, einmal weil es sich um einen sehr seltenen pathologisch-anatomischen Befund und um eine seltene Wirkung dieses Befundes handelt, zweitens weil er zugleich geeignet ist, zur Lösung der allgemein pathologischen Frage von dem Entstehungsort des epileptischen Anfalls beizutragen.

Die Krankengeschichte ist sehr kurz: Das 45 Jahre alte Fräulein S. wurde am 13. November 1912 in das Weißenseer Krankenhaus in bewußtlosem Zustande von der Polizei eingeliefert. Sie blieb bewußtlos, und nur auf kurze Zeit schien sie etwas auf Anrufe zu reagieren.

Sie zeigte häufig sich wiederbolende, stundenweis alle 5-10 Minuten wiederkehrende epileptisehe Anfälle von Jackso nschem Typus. Diese Anfälle begannen immer im linken Facialis zugleich mit Drehung der Augen und des Kopfes nach links, gingen von da auf den linken Arm und von da auf das linke Bein über, um dann meist allgemein zu werden.

Die später vernommenen Angehörigen geben an, daß über frühere Krämpfe ihnen nichts bekannt sei.

Der Zustand schien von vornherein fast aussichtslos. Die Kranke wurde zunächst mit Excitantien und Narkoticis behandelt. Als wir beide die Kranke am 16. XI. zuerst sahen, war sie völlig benommen. Die Pupillen waren different und reagierten nicht auf Lichteinfall. Außerhalb der Anfälle war eine Parese des linken Armes und auch des linken Beines festzustellen. Indessen wurde trm und Bein doch bei den Reaktionen auf schmerzhafte Reize noch bewegt. Die Sehnenreflexe links waren gesteigert; Fußklonus bestand nicht, ebenso wenig Babinski. Bei der Beobachtung zweier Krampfanfälle fiel auf, daß diese mit einem mehrere Sekunden lang dauernden tonischen Stadium im linken Facialis begannen; während dann dieser Tonus auf den Arm fortschritt, begannen erst klonische Zuckungen im Facialis dann auch klonische Zuckungen im Arm und auch im Bein war ein längeres tonisches Vorstadium von den klonischen Krämpfen, die hier überhaupt schwach waren, zu unterscheiden.

Es wurde uns bestätigt, daß alle Anfälle in der gleichen Weise verlaufen waren.

Trotz des desolaten Zustandes — es bestand bereits Lungenödem - erschien eine Operation doch noch gerechtfertigt, und zwar wurden, entsprechend dem Beginn 
der Krämpfe im Facialis etwa die beiden unteren Drittel der Zentralregion freigelegt (Bildung eines Hautknochenlappens). Nach Aufklappen eines entsprechenden Duralappens erschien die Hirnoberfläche hier intakt, die Probepunktion und -excision brachte kein Ergebnis. Der Hautlappen wurde wieder vernäht, die Patientin erlag einige Stunden nach der Operation.

Die Autopsie brachte nun ein sehr merkwürdiges Ergebnis: Bei der Herausnahme erschien die Pia im Bereiche des hinteren Drittels der ersten Stirnwindung und des hinteren Teiles der vorderen Zentralwindung etwas blutig suffundiert, indessen so wenig, daß dies auch Operationsfolge sein konnte. Immerhin war auffallend, daß diese Suffusion außerhalb der Trepanationsöffnung lag. Das Gehirn wurde nun im ganzen in Formol gehärtet. Danach wurde an der Stelle, die am meisten verändert erschien - ungefähr an der vorcleren Grenze des hintersten Viertels der ersten Frontalwindung - ein Querschnitt angelegt. Es zeigten sich hier auch in der Rinde diffuse und zerstreute Blutungen auf einem Gebiet von auf dem Querschnitt noch nicht Markstückgröße. Die Blutungen sahen makroskopisch aus wie eine hämorrhagische Encephalitis. Mikroskopisch ergaben sich jedoch keine Zeichen einer Entzündung, sondern es handelte sich nur um einfache Blutaustritte in das Gewebe.

Wir wollten diesen Befund erst auf Rechnung der Operation setzen; als wir aber noch einige Frontalschnitte anlegten, um die Ausdehnung dieser Blutungen nachzusehen, kamen wir ein wenig hinter der Fissura praecentralis, also im vordersten Gebiete der vorderen Zentralwindung, auf einen offenbar präformierten mit Blut gefüllten Hohlraum von etwa $8 \mathrm{~mm}$ Durchmesser. In Tafel VII, Fig. 1 ist dieser Hohlraum mit T bezeichnet. Er liegt z. T. in der Rinde, z. T. subcortical, und zwar, wie die Figur zeigt, etwa auf der Grenze zwischen medialem und mittlerem Drittel der mediolateralen Ausdehnung der Windung. Medial von diesem Hohlraum liegt im Gewebe noch freies Blut (B).

Die mikroskopische Untersuchung ergab, daß der Hohlraum ein aneurysmatisch ausgedehntes Gefäß darstellte. Medial von diesem Hohlraum befindet sich ein Konvolut von Gefäßschlingen und Hohlräumen, die zum Teil noch mit Blut gefüllt sind und ersichtlich ein Angioma cavernosum darstellen (Tafel VII, Fig. $2 a$ ), die aher nur ein viel kleineres Territorium einnehmen, als der große Hohlraum. Der große Hohlraum ist von einer eigenen dünnen Wandmembran umgeben, die auf der $\mathrm{Ab}$ bildung da besonders deutlich ist, wo sie sich von dem umgebenden $\mathrm{Ge}$ webe abgehoben hat (Tafel VII, Fig. 2c). Im Bereiche des unteren medialen Quadranten (Tafel VII, Fig. 2d) ist diese Wand jedoch geplatzt und von hier sind Blutungen in das Gewebe erfolgt (Tafel VII, Fig. 2b), die sich dann in der obenerwähnten Ausdehnung ausgebreitet haben ${ }^{1}$ ).

1) Wan wird daran denken müssen, da $B$ solche Blutgefäßgeschwülste häufiger die Quelle dunkler Blutungen bei jugendlichen Individuen sind. Es war ja auch 
Was die allgemeine klinische Seite des Falles betrifft, so handelt es sich somit um ein unter der Oberfläche des Gehirns liegendes A ngio m von im ganzen $1 \mathrm{~cm}$ Durchmesser, wovon der eigentliche Tumor nur einen kleinen Teil ausmacht, der Hauptteil vielmehr aus einem aneurysmaähnlichen Blutsack besteht. Wir wollen auf die Literatur dieser Geschwulst, auf welche in den letzten Jahren von Astwazatu-

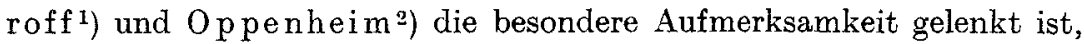
um so weniger eingehen, als vor kurzem in dieser Zeitschrift ${ }^{3}$ ) von J. $S$ wease y Powers ein Fall genauer beschrieben worden ist. In unserem Fall war von einer genaueren Diagnose mangels einer Anamnese gar keine Rede. Übrigens scheint die Patientin bis kurz vor der Aufnahme völlig gesund gewesen $\mathrm{zu}$ sein, so daß uns wahrscheinlich auch eine Anamnese nichts genützt hätte. Es ergab sich aus den Jacksonschen Krämpfen nur die praktische Notwendigkeit der Trepanation. Bemerkenswert ist, daß diese Trepanation an einer nicht ganz richtigen Stelle ausgeführt werden mußte, wenn man - wie das allgemein üblich - das primär-krampfende Zentrum freilegte. Die Krämpfe gingen vom Facialis aus, und der Tumor saß etwa zwischen Bein- und Armregion. Das hat vielleicht darin seinen Grund, daß der Tumor an der vorderen Grenze der vorderen Zentralwindung saß und die von ihm ausgehende Blutung sich wesentlich nach vorn ausbreitete, während die eigentlichen motorischen Zentren wesentlich hinter dem Tumor, ziemlich dicht an der Rolandoschen Furche liegen, so daß also die Reizwirkung keine unmittelbare war, sondern der Reiz erst fortgeleitet wurde. Praktisch erscheint diese mangelnde U̇bereinstimmung zwischen Tumorsitz und Beginn der Krämpfe verhängnisvoll, weil man keine Mittel besitzt, diesen Fehler zu korrigieren, und man unmöglich immer die ganze motorische Region freilegen kann. Für unseren Fall sei speziell noch hervorgehoben, daß trotz der Nähe des Tumors zum Beinzentrum der Babinskische Reflex negativ war, so daß wir um so weniger einen Herd in der Beinregion annehmen durften. Freilich wäre auch bei der

in unserem Falle ein halber Zufall, daß die kleine Geschwulst in der umfangreichen Blutung überhaupt entdeckt wurde. Bleiben solehe Fälle am Leben, ohne daß der Tumor weitere Fortschritte macht, so wird natürlich niemand einen Tumor diagnostizieren können, aber möglich wird er doch sein. Wir erinnern hier z. B. an einen von Lewandowsk y und Stadel mann (Journ. f. Psychol. u. Neurol. 9, 249. 1908) beschriebenen Fall, wo bei einem jungen Menschen mit Staungspapille durch Hirnpunktion ca. $60 \mathrm{ccm}$ Blut aus dem Occipitallappen entleert wurden, und der Mann dureh diesen Eingriff gerettet wurde (der Mann ist bis heute völlig gesund geblieben). In diesem und ähnlichen Fällen wird man intra vitam immerhin an eine Blutung aus einem Gefäßtumor denken dürfen und bei einer eventuellen Autopsie recht sorgfältig nach einem solchen suchen müssen.

1) Astwazaturoff, Frankfurter Zeitschr. f. Pathol. 8, 481, H. 3.

2) Op penheim, Neurol. Centralbl. 1913, 3.

3) Zeitschr. f. d. ges. Neur. u Psych. Orig. 16, 487. 1913. 
Freilegung der richtigen Stelle in unserem Falle nicht viel gewonnen gewesen, da die Erkennung des Tumors wahrscheinlich nicht gelungen wäre, weil er zu klein war, und auch nirgends die Oberfläche des Gehirns erreichte. Die blutige Suffusion der Pia in der oberflächlichen Gehirnschicht hätte aller Wahrscheinlichkeit nach zur Annahme einer einfachen Rindenblutung geführt, selbst wenn unwahrscheinlicherweise gerade das beschriebene aneurysmaähnliche Gebilde eröffnet worden wäre, hätte man wohl nicht an einen Tumor gedacht, da die Blut ung aus dem Tumor den ganz überwiegenden Teil der pathologischen Veränderungen darstellte.

Von allgemein pathologischer Seite ist der Fall bemerkenswert durch das ausgesprochen tonische Vorstaclium der Jacksonschen Krämpfe. Es ist bekanntlich von Ziehen und Binswanger ${ }^{1}$ ) die Behauptung aufgestellt worden, daß die klonische Komponente des epileptischen Anfalles in der Rinde, die tonische subcortical, d. h. im Hirnstamm entsteht. Die experimentellen Grundlagen dieser Anschauung sind vor längerer Zeit bereits von dem einen von uns widerlegt worden ${ }^{2}$ ), der auf Grund seiner Versuche sich der Unverrichtschen Anschauung anschloß, daß der ganze epileptische Krampf wesentlich in der Rinde entsteht und abläuft und jedenfalls die scharfe Trennung der klonischen von der tonischen Componente bestritt. Klinisch beruht die Ziehen-Binswangersche Aufstellung wesentlich auf einem Gegensatz der ,großen" epileptischen Anfälle und der Jacksonschen insofern, als die letzteren mit einem Klonus anfangen sollen, die ersteren mit einem Tonus. Nun ist es aber sehr merkwürdig, daß eine Reihe von Autoren, Gowers, Wernicke, Oppenheim, einen solchen Gegensatz nicht betonen. Redlich, der diesen Gegensatz anerkennt, sagt, daß auch beim Jacksonschen Anfall ein tonisches Vorstadium nicht allzu selten ist. Wir selbst haben in den von uns beobachteten Fällen bisher immer einen klonischen Beginn gesehen, wenn man nicht eine kurze tonische, etwa wie eine verlängerte willkürliche aussehende Bewegung, welche dem Klonus oft vorausgeht, als tonischen Krampf rechnen will. Wir müssen daher klinisch ein so ausgesprochenes tonisches Vorstadium wie in dem beobachteten Falle auch für eine Ausnahme halten. Um so bemerkenswerter ist nun der pathologische Befund eines corticalen Tumors bzw. corticaler Blutung in einem solchen Falle und das Fehlen jedes Befundes im Hirnstamm ${ }^{3}$ ). Dadurch wird bewiesen,

1) Vgl. genauer bei Binswanger, Die Epilepsie, 2. Aufl. Wien-Leipzig 1913. S. 51.

2) Lewandowsky, Funktionen des Nervensystems. Jena 1904. S. 250 und 251. Weil diese Versuche nicht in einer besonderen Arbeit publiziert wurden, sind sie allgemein übersehen worden.

${ }^{3}$ ) W. Bra un hat im Krankenhaus Friedrichshain, wie er uns mitteilt, jüngst einen Fall nach Schädeltrauma beobachtet, dessen Krämpfe genau so verliefen, 
340 M. Lewandowsky und F. Selberg: Über Jacksonsche Krämpfe usw.

daß auch das tonische sich in Form der Jacksonschen Krämpfe ausbreitende Vorstadium des epileptischen Krampfes cortical bedingt sein kann, und damit fällt auch jede Nötigung fort, für den großen epileptischen Anfall eine subcorticale Komponente anzunehmen ${ }^{1}$ ).

wie die in unserem Fall. Die Autopsie am formolgehärteten Gehirn ergab in diesem Fall nur eine subdurale Blutung über dem Stirnhirn der entsprechenden (contralateralen) Seite.

1) Daß beim Hund auch bei Fehlen des Großhirns tonische Krämpfe zustande kommen können, wie Rothmann beobachtete, beweist nicht einmal für den epileptischen Krampf des Hundes etwas. Außerdem aber sind die Ergebnisse krampferzeugender Reizungen der Rinde schon beim Affen andere als beim Hund (Lewandowsky l.c.), so daß auch ein Schluß rom Hund auf den Menschen unzulässig erscheint. 